\title{
Multi-Bandwidth Frequency Selective Surfaces For Near Infrared Filtering: Design and Optimization
}

\author{
Tom Cwik*a, Salvador Fernandez ${ }^{\mathrm{b}}$, A. Ksendzova, Clayton C. La Bawa, Paul D. Makerª, \\ Richard E. Muller" \\ aJet Propulsion Laboratory, California Institute of Technology, 4800 Oak Grove Dr., Pasadena, \\ CA 91109 \\ ${ }^{b}$ Ciencia, Inc., 111 Roberts St., Suite C, East Hartford, CT 06108
}

\section{INTRODUCTION}

Frequency selective surfaces are widely used in the microwave and millimeter wave regions of the spectrum for filtering signals. They are used in telecommunication systems for multi-frequency operation or in instrument detectors for spectroscopy. The frequency selective surface operation depends on a periodic array of elements resonating at prescribed wavelengths producing a filter response. The size of the elements is on the order of half the electrical wavelength, and the array period is typically less than a wavelength for efficient operation. When operating in the optical region, diffraction gratings are used for filtering. In this regime the period of the grating may be several wavelengths producing multiple orders of light in reflection or transmission.

In regions between these bands (specifically in the infrared band) frequency selective filters consisting of patterned metal layers fabricated using electron beam lithography are beginning to be developed. The operation is completely analogous to surfaces made in the microwave and millimeter wave region except for the choice of materials used and the fabrication process. In addition, the lithography process allows an arbitrary distribution of patterns corresponding to resonances at various wavelengths to be produced.

The design of sub-millimeter filters follows the design methods used in the microwave region. Exacting modal matching, integral equation or finite element methods can be used for design. A major difference though is the introduction of material parameters and thicknesses that may not be important in longer wavelength designs. This paper describes the design of multibandwidth filters operating in the 1-5 micrometer wavelength range. This work follows on a previous design $[1,2]$. In this paper extensions based on further optimization and an examination of the specific shape of the element in the periodic cell will be reported. Results from the design, manufacture and test of linear wedge filters built using microlithographic techniques and used in spectral imaging applications will be presented.

\section{METHODS FOR DESIGN AND ANALYIS OF THE ARRAY}

Two different methods are applied in the analysis and design of the frequency selective arrays. The first uses a moment method solution to the electric field integral equation formulation to calculate transmission and reflection coefficients of the filter [3]. In this code (FSS) piecewise basis functions are used to model the metal surface and an impedance boundary 
The opportunity to specify a particular wavelength versus position sequence to emphasize certain spectral regions is very attractive to both commercial and government agency users.

The electron micrograph of a sample before metal deposition is shown in Fig. la and a micrograph of a completed mesh filter is shown in Fig. 1b. A wide area $\left(1 \mathrm{~cm}^{2}\right)$ single wavelength filter has been produced and measured. In Figure 2 the comparison of the calculated (using FSS) and measured transmittance curves is shown. The measurements were performed using a Beckman IR4250 Spectrophotometer in a $\mathrm{f} / 10$ beam [6]. The calculated and measured curves agree well. The filter has very good rejection at wavelengths longer than the center of the pass band. There is an undesirable transmission band on the shorter wavelength side that turned out to be somewhat larger than predicted.

\section{ACKNOWLEDGMENT}

The research described in this paper was carried out by the Jet Propulsion Laboratory, California Institute of Technology, under contract with the National Aeronautics and Space Administration. Partial sponsorship of this research lies with the Department of Defense Ballistic Missile Defense Organization under the auspices of a Small Business Technology Transfer Program managed by Ciencia, Incorporated of East Hartford, CT. The supercomputer used in this investigation was provided by funding from the NASA Offices of Earth Science, Aeronautics, and Space Science.
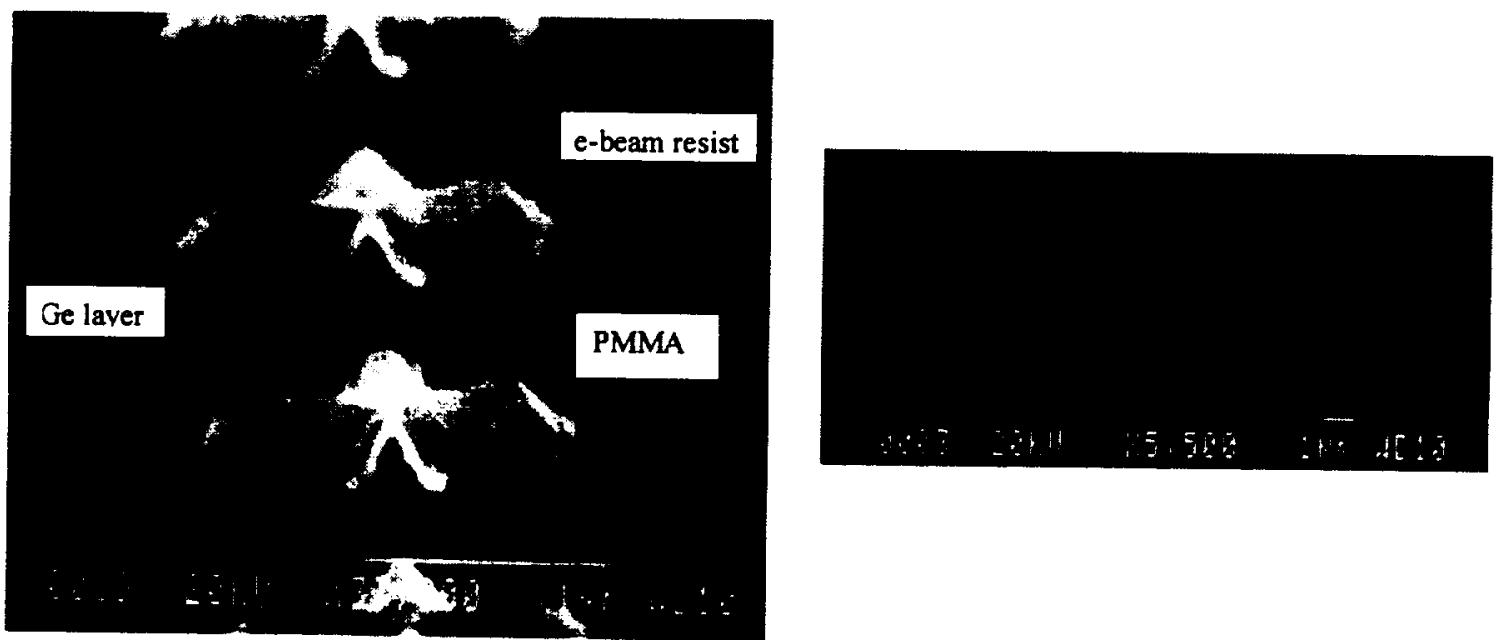

Figure 1. Micrograph of sample periodic screen before metal deposition (left). A portion of the completed mesh filter (right). 


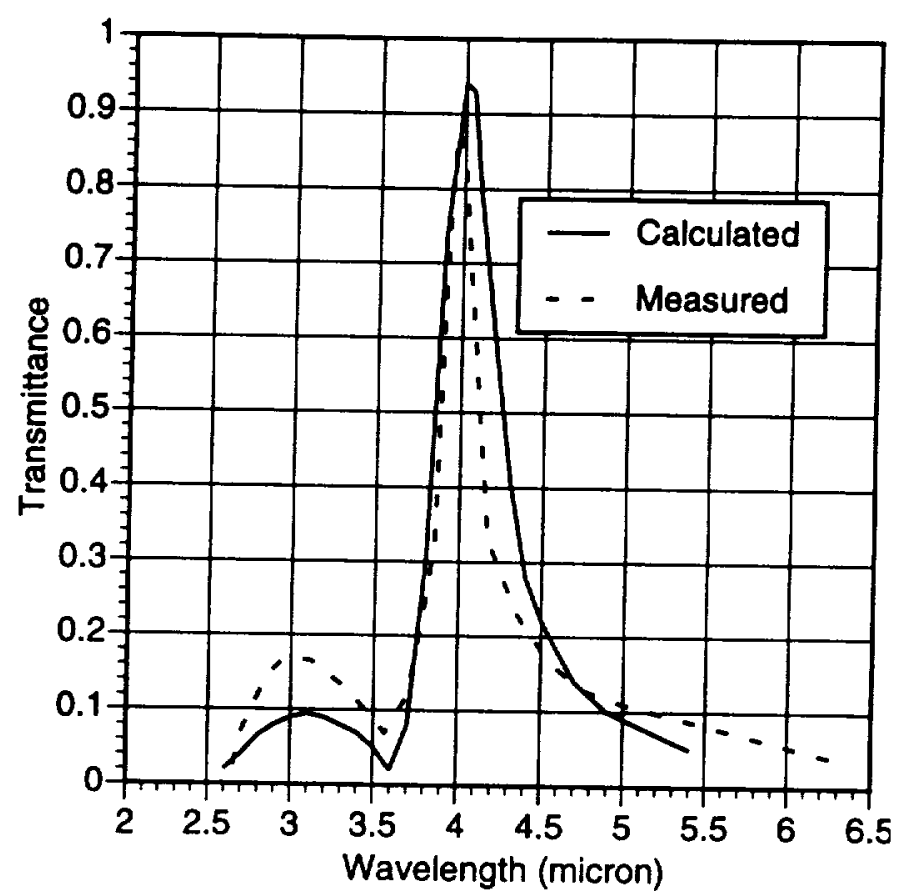

Figure 2. Calculated and measured single filter transmittance.

\section{REFERENCES}

1. Ksendzov, A., Fernandez, S., Cwik, T., La Baw, C. Maker, P. and Muller R., "Wedge Filters for Spectral Imaging in the Near IR Using Metal Grids, Proceedings Infrared Astronomical Instrumentation. Vol 3354, 1998

2. Cwik T, Fernandez, S., Ksendzova, A., La Baw, C. Maker, P., and Muller, R. "Design of multi-bandwidth frequency selective surfaces for near infrared filtering," SPIE's 43rd Annual Meeting on Optical Science, Engineering, and Instrumentation, San Diego, CA July 19-24, CA 1998.

3. Cwik T. and Mittra R., "Scattering from a periodic array of free-standing arbitrarily shaped perfectly conducting or resistive patches", IEEE Trans. Antennas Propag., vol. AP-35, no. 11, pp. 1226-1233, Nov. 1987.

4. McGrath, D. PARANA Version 2, Users Guide, USAF Phillips Laboratory, May 1997.

5. Zuffada, C. and T. Cwik, "Synthesis of Novel All-Dielectric grating Filters Using Genetic Algorithms," IEEE Trans. Antennas Propag., Vol. 46, No. 5, pp.657-663, May. 1998.

6. La Baw, C. "Measuring Spatially Varying Transmittance of a Filter", NASA Tech Briefs, pg. 66, September 1992. 